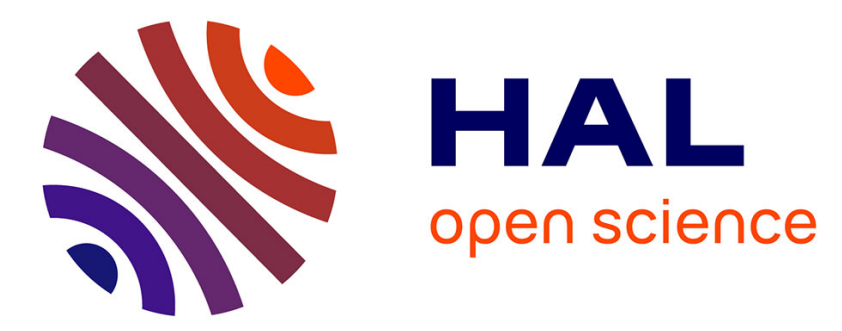

\title{
Left-handed optical radiation torque
}

Davit Hakobyan, Etienne Brasselet

\section{To cite this version:}

Davit Hakobyan, Etienne Brasselet. Left-handed optical radiation torque. Nature Photonics, 2014, 8

(8), pp.610-614. 10.1038/nphoton.2014.142 . hal-01061787

\section{HAL Id: hal-01061787 \\ https://hal.science/hal-01061787}

Submitted on 23 Feb 2016

HAL is a multi-disciplinary open access archive for the deposit and dissemination of scientific research documents, whether they are published or not. The documents may come from teaching and research institutions in France or abroad, or from public or private research centers.
L'archive ouverte pluridisciplinaire HAL, est destinée au dépôt et à la diffusion de documents scientifiques de niveau recherche, publiés ou non, émanant des établissements d'enseignement et de recherche français ou étrangers, des laboratoires publics ou privés.

\section{다)(1) $(5$}

Distributed under a Creative Commons Attribution - NonCommercial| 4.0 International 


\title{
Left-handed optical radiation torque
}

\author{
Davit Hakobyan and Etienne Brasselet ${ }^{\star}$
}

\begin{abstract}
Optical forces and torques are two mechanical degrees of freedom available to manipulate matter, and form the basis of optical tweezing strategies ${ }^{1,2}$. In contrast to the Keplerian intuition that objects should be pushed downstream an incident photon flux, the concept of 'negative' optical forces has recently been described ${ }^{3,4}$ and has triggered many developments $^{5-14}$. Here, we report on the counterintuitive angular analogue of negative optical forces by demonstrating that circularly polarized Gaussian light beams give rise to torque with opposite sign to that of the incident optical angular momentum. Such a 'left-handed' mechanical effect is demonstrated by the use of an inhomogeneous and anisotropic transparent macroscopic medium. Practical difficulties associated with the direct observation of optically induced spinning of a macroscopic object are circumvented via the rotational Doppler effect ${ }^{15,16}$. These results shed light on spin-orbit optomechanics and equip the left-handed optomechanical toolbox with angular features.
\end{abstract}

The redirection of a photon flux by a material system is essen tially associated with non conservative optical radiation forces. In particular, negative optical forces occur when the linear momentum of light experiences a net forward scattering that depends on the structure of the incident electromagnetic fields, the properties of the objects and their surrounding environment ${ }^{11}$. Quite naturally, because scattering is a universal feature common to all waves, the concept of negative forces is also found in acoustics ${ }^{17-19}$. Intriguingly, its angular counterpart has not yet emerged. This corresponds to a light field exerting an optical radiation torque (ORT) on matter, where the direction of the torque is reversed with respect to that of the incident angular momentum assumed to be at play to produce the ORT. We shall refer to such a reverse angular optomechanical effect as 'left handed' instead of 'negative' to avoid confusion, as it may occur either with $\tau_{z}>0$ or $\tau_{z}<0$, where $\tau_{z}$ is the projection of the ORT along $z$ that defines the pro pagation direction of the light. Accordingly, we will refer to 'right handed' for the intuitive common situation. Here, we report the observation of left handed ORT for the case of a circularly polarized Gaussian light beam carrying an angular momentum per photon of $\sigma \hbar$ along its propagation direction $z(\sigma= \pm 1, \hbar$ is the reduced Planck constant), namely $\sigma \tau_{z}<0$, whatever $\sigma$.

The non trivial nature of left handed ORT is exemplified by recalling the two types of light matter interaction processes leading to ORT that are driven by spin angular momentum. The first is the dissipative case where the exchange of angular momen tum is driven by absorption, as experimentally reported in ref. 20 . By considering an absorbing optically isotropic object characterized by an overall power attenuation coefficient of $0 \leq \varepsilon \leq 1$, the angular momentum balance gives $\tau_{z}=\sigma \varepsilon \hbar$ (Fig. 1a). Because $\sigma \tau_{z}=\varepsilon \hbar \geq 0$, left handed ORT cannot occur. The second case refers to non dissipative exchange of angular momentum involving a birefringent medium, as demonstrated in 1936 using a centimetre sized crystalline half wave plate ${ }^{21}$, inspiring significant advances in down sizing down to the micrometre scale ${ }^{22}$. The angular momentum balance gives $\tau_{z}=\sigma(1-\cos \Delta) \hbar$, where $\Delta$ is the birefringent phase retardation of the wave plate (Fig. 1b). As $\sigma \tau_{z}=(1-\cos \Delta) \hbar \geq 0$, left handed ORT is also prohibited.

Our idea to achieve left handed ORT consists of coupling the incident spin angular momentum to the spatial degrees of freedom of the light field, namely orbital angular momentum. Indeed, if ORT is dominated by the orbital contribution rather than that of the spin, reversing the angular momentum balance becomes possible. Because such spin orbit light scattering basically takes place in optically inhomogeneous and anisotropic transparent media, we propose, for the purpose of demonstration, to restrict the present study to space variant uniaxial slabs with constant birefrin gent phase retardation $\Delta$ and optical axis orientation angle $\psi$ of the form $\psi(\phi)=q \phi$, where $q$ is the half integer and $\phi$ the polar angle in the plane of the slab. When $\Delta=\pi$, a collimated circularly polarized Gaussian beam carrying an incident spin angular momentum per photon $s_{z}^{\text {in }}=\sigma \hbar$ that impinges on axis onto the azimuthally pat terned slab will emerge from it with a total angular momentum per photon of $j_{z}^{\text {out }}=s_{z}^{\text {out }}+l_{z}^{\text {out }}$, where $s_{z}^{\text {out }}=-\sigma \hbar$ and $l_{z}^{\text {out }}=2 \sigma q \hbar$ are respectively the spin and orbital contributions ${ }^{23}$. The angular momentum balance $\tau_{z}=j_{z}^{\text {in }}-j_{z}^{\text {out }}$ thus gives

$$
\tau_{z}=2 \sigma(1-q) \hbar
$$

Because $\sigma \tau_{z}=2(1-q) \hbar$ whatever $\sigma$, left handed ORT is expected when $q \geq 3 / 2$, whereas right handed ORT takes place when $q \leq$ $1 / 2$, as shown in Fig. 1c. In addition, the particular case of $q=1$ corresponds to vanishing ORT, as expected from the rotational invariance around $z$.

In practice we used azimuthally patterned birefringent disk shaped glass slabs with $q=(1 / 2,1,3 / 2)$ and $\Delta=\pi$ at a wavelength of $532 \mathrm{~nm}$. The spatial distribution of the slow axis is depicted in Fig. 2 (first column). The samples were fabricated by femtosecond laser writing of self assembled nanogratings in silica glass ${ }^{24}$, and the induced form birefringence patterns were achieved by control ling the writing parameters, in particular the polarization azimuth of the writing beam ${ }^{25}$. Their polariscopic optical characterization at a wavelength of $532 \mathrm{~nm}$ is summarized in Fig. 2, where crossed linear polarizer imaging (second column) emphasizes the azimuthal patterning of the optical axis, and crossed and parallel circular polarizer imaging (third and fourth columns) present the uniform birefringent phase retardation $\Delta=\pi$. See also the 'Materials and Methods' section.

A straightforward experimental approach for observing the optical torque would consist of direct observation of the light induced spinning of a free to rotate sample around the $z$ axis, the left handed nature of the ORT being retrieved from a sense of rotation opposite that prescribed by the incident spin angular momentum. However, considering a transfer of $\hbar$ angular momen tum per photon, a light beam with power $P$ and angular frequency $\omega$ produces a torque of magnitude $\Gamma=P / \omega$, so $\Gamma \approx 1 \times 10^{-16} \mathrm{~N} \mathrm{~m}$ 

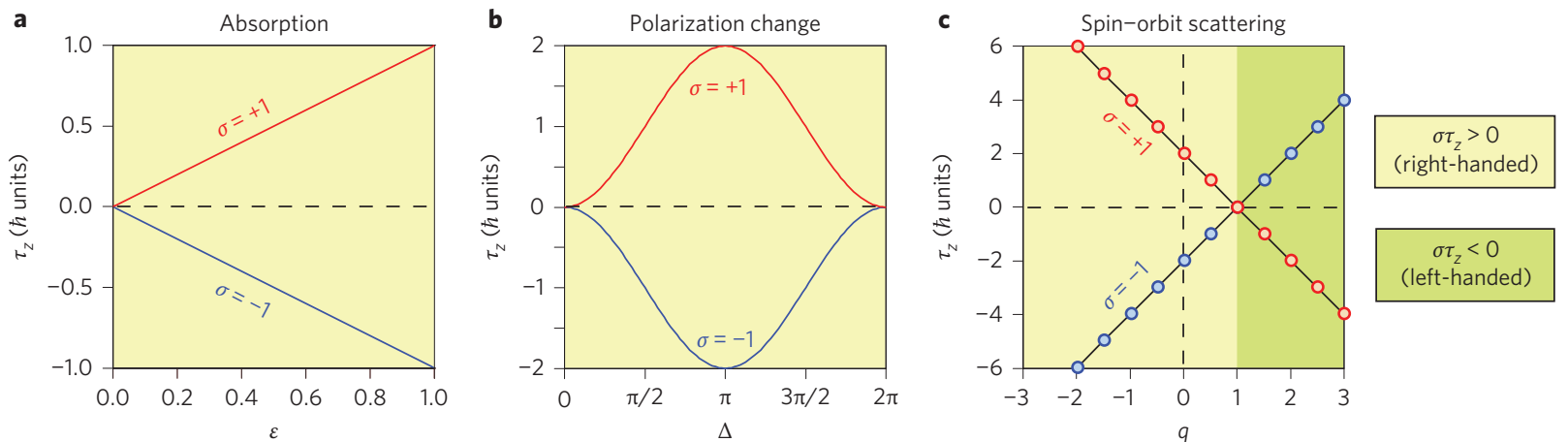

Figure 1 | Comparison of the various kinds of ORT produced by circularly polarized Gaussian beams. a, Absorption characterized with overall power-attenuation coefficient $0 \leq \varepsilon \leq 1$. b. Polarization change associated with a birefringent phase retardation $0 \leq \Delta \leq 2 \pi$. c, Optical spin-orbit interaction of light mediated by an azimuthally variant half-wave plate of half-integer order $q$ (see text for details).
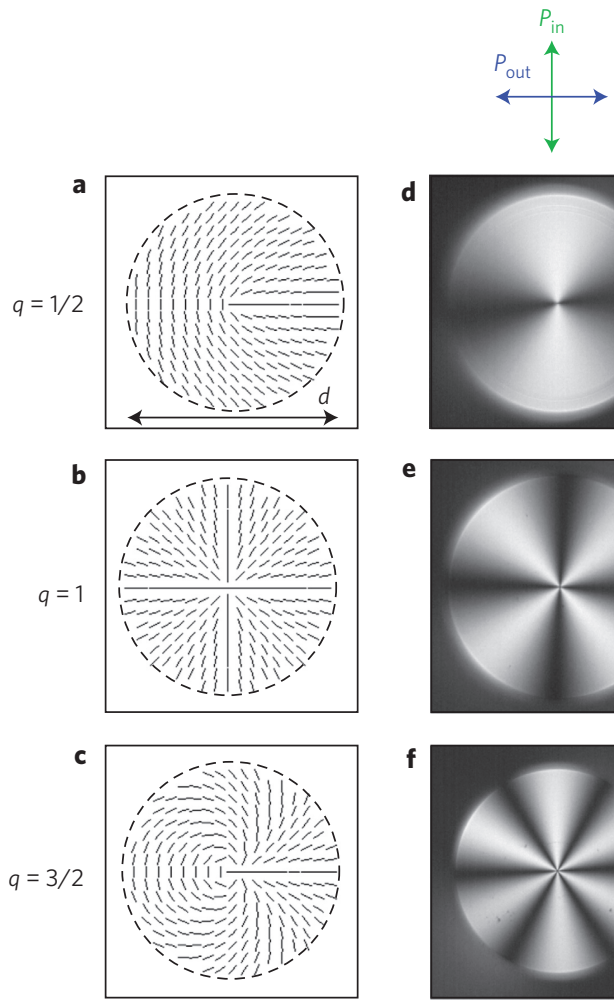
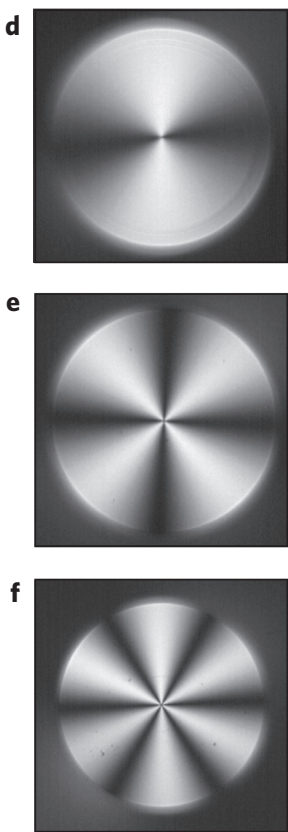
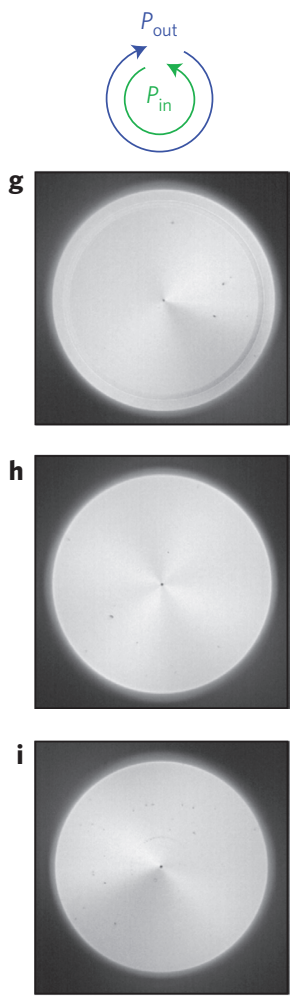
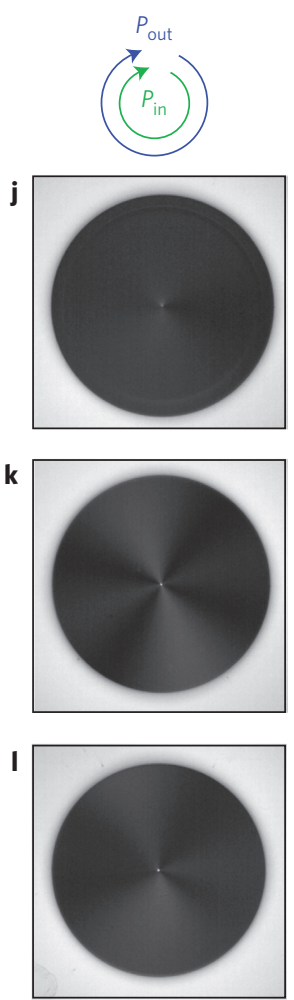

Figure 2 | Spin-orbit light scattering samples. a-c, Slow-axis spatial distribution of the form-birefringent nanostructured glass plates with $q=1 / 2,1$ and $3 / 2$, where segments indicate local orientation. $\mathbf{d}-\mathbf{I}$, Polariscopic analysis performed by optical imaging between crossed linear polarizers (d-f), crossed circular polarizers ( $\mathbf{g}-\mathbf{i})$ and parallel circular polarizers $(\mathbf{j}-\mathbf{I})$ under incoherent spatially extended illumination at a wavelength of $532 \mathrm{~nm}$. $P_{\text {in }}$ and $P_{\text {out }}$ denote the input and output polarization states of light. The structured area has a circular cross-section with diameter $d=4 \mathrm{~mm}$.

under $1 \mathrm{~W}$ visible light illumination. Then, assuming free rotation in an inviscid medium one finds a rotation frequency $v$ that depends on the illumination time $T$ following $v(T)=\Gamma T /\left(\pi m R^{2}\right)$, where $m$ is the mass of the slab. According to the sample parameters (the sample is structured over an area of diameter $d=4 \mathrm{~mm}$ centred on a disk shaped glass substrate of radius $R=12.7 \mathrm{~mm}$, thickness $H=3 \mathrm{~mm}$ and density $\rho=2.5 \times 10^{3} \mathrm{~kg} \mathrm{~m}^{-3}$ ) one typically obtains $v=1 \mathrm{mHz}$ after a three month illumination at $P=1 \mathrm{~W}$, which clearly prevents a realistic experimental implementation.

The practical difficulty of observing the mechanical consequence of the spin orbit scattering process on matter is circumvented by probing the mechanical effect of the ORT on the light itself. This is implemented using a rotational Doppler experiment ${ }^{15,16}$ by rotat ing the slab at controlled angular velocity $\Omega$ around the $z$ axis. Non zero ORT therefore causes a frequency shift $\delta \omega$ for the output light, whose expression can be derived from energy conservation, namely $w+\hbar \delta \omega=0$ where $w=\tau_{z} \Omega$ is the work per photon produced on the slab. This gives

$$
\delta \omega=2 \sigma \Omega(q-1)
$$

Because $\tau_{z}=\hbar \delta \omega / \Omega$, we stress that the determination of the rotational Doppler frequency shift is actually an ORT measurement.

The experiment was carried out by observing the intensity pat terns that result from the non collinear superposition of the output light field and a reference Gaussian beam with angular fre quencies $\omega+\delta \omega$ and $\omega$, respectively, as outlined in Fig. $3 \mathrm{a}$. Indeed, we observe $2 q$ fork interference patterns that are 
reminiscent of the optical phase singularity with topological charge $2 q \sigma$ emerging from the sample ${ }^{23}$, as illustrated by the snapshots shown in Fig. $3 \mathrm{~b} d$ for $q=(1 / 2,1,3 / 2)$. The determination of $\delta \omega$ is achieved by analysing the dynamics of these intensity patterns, denoted $I(x, y, t)$, with time $t$. Indeed, by constructing spatiotem poral interferograms $I\left(x=x_{0}, y, t\right)$ we obtain fringing patterns that may or may not drift along the $y$ axis depending on $q, \sigma$ and $\Omega$. This is illustrated in Fig. $3 \mathrm{e}$ g, for $q=(1 / 2,1,3 / 2)$ when $\sigma \Omega>0$.

The sign of the latter drift gives access to the sign of $\delta \omega$ from a mere visual inspection. In other words, with our set up, when $\sigma \Omega>0$, a drift towards $y<0$ refers to $\delta \omega<0$ (Fig. 3e), whereas a drift towards $y>0$ indicates $\delta \omega>0$ (Fig. $3 \mathrm{~g}$ ), and no drift implies $\delta \omega=0$ (Fig. 3f) (Supplementary Section 1). Because $\sigma \tau_{z}=\hbar \delta \omega / \sigma \Omega$, our results indicate that $\sigma \tau_{z}<0$ when $q=3 / 2$, hence demonstrating that left handed ORT takes place.

Regarding the magnitude of $\delta \omega$, we define the time dependent quantity $\bar{I}(t)=\int I\left(x_{0}, y, t\right) \mathrm{d} y-\left\langle\int I\left(x_{0}, y, t\right) \mathrm{d} y\right\rangle_{t}$ (Fig. $\left.4 \mathrm{a}\right)$, where $\langle\cdot\rangle_{t}$ holds for time averaging, where the spectrum exhibits a well defined peak at frequency $|\delta \omega| / 2 \pi$ (Fig. 4b), as demonstrated in Supplementary Section 2. The dependence of $\delta \omega$ as a function of $\Omega$ is shown in Fig. $4 \mathrm{c}, \mathrm{d}$, corresponding to the range $|\delta \omega| / \omega=$ $10^{-17} 10^{-16}$ for $q=(1 / 2,3 / 2)$ and $\sigma= \pm 1$. In Fig. $4 \mathrm{c}, \mathrm{d}$, the solid lines refer to the theoretical results given by equation(2), from which we can infer the excellent agreement between the experimen tal data and theoretical predictions. We thus obtain the precise experimental determination of both the sign and magnitude of the rotational Doppler frequency shift, thereby allowing the quanti tative identification of left handed ORT.

Next we address the question of whether left handed ORT may occur for $\Delta \neq \pi$. In this case the incident light field emerges from the slab with a total angular momentum per photon of $j_{z}^{\text {out }}=\sigma\left[\cos \Delta+2 q \sin ^{2}(\Delta / 2)\right] \hbar$ (Supplementary Section 3) from which results an ORT of

$$
\tau_{z}=2 \sigma(1-q) \sin ^{2}(\Delta / 2) \hbar
$$

Previous conclusions are therefore unaltered whatever the value of $\Delta$, up to a factor of $\sin ^{2}(\Delta / 2)$ for the ORT magnitude. Experimentally, this situation is explored by detuning the incident wavelength from $532 \mathrm{~nm}$, setting it at $632.8 \mathrm{~nm}$, so $\Delta=(532 / 632.8) \pi$ (that is, $0.84 \pi)$. The results are displayed in Fig. 5, where the rotational Doppler effect is assessed independently for the two output light field components with opposite helicities $\pm \sigma$ and $q=(1 / 2,1,3 / 2)$. This is done by using a circularly polarized reference beam with helicity $\sigma_{\text {ref }}=\sigma$ (Fig. 5b d) and $\sigma_{\text {ref }}=\sigma$ (Fig. 5e g), respectively. We conclude from Fig. 5b d that photons whose helicity is unchanged did not produce ORT. In con trast, helicity flipped photons give rise to right handed, zero or left handed ORT depending on $q$, as shown in Fig. $5 \mathrm{e}$ g. Noticeably, the factor $f=\sin ^{2}(\Delta / 2)$ in equation (3) corresponds to the fraction of output photons that produce work on the sample (Supplementary Section 3). In addition, because the energy conservation condition per photon should be written 'per working photon', hence $\tau_{z} \Omega+$ $f \hbar \delta \omega=0$, the predicted magnitude of $\delta \omega$ is unchanged with respect to the case $\Delta=\pi$. This has been quantitatively verified and can be qualitatively grasped from a comparison of Fig. $3 \mathrm{e} g$ and Fig. $5 \mathrm{e}$ g. We thus generalize the proposed concept of left handed ORT to arbitrary values of birefringent phase retardation.

The above results remain valid for any superposition of left and right handed circularly polarized Gaussian beams. Indeed, account ing for an electric field of the form $\alpha G_{+1}+\beta G_{-1}$ where $(\alpha, \beta) \in \mathbb{C}^{2}$ and $G_{\sigma}$ refers to a Gaussian field with helicity $\sigma$, one obtains $s_{z}^{\text {in }} \tau_{z}=2\left[\left(|\alpha|^{2}-|\beta|^{2}\right) /\left(|\alpha|^{2}+|\beta|^{2}\right)\right]^{2}(1-q) \sin ^{2}(\Delta / 2) \hbar^{2}, \quad$ which leaves the previous conclusions unchanged. Namely, $s_{z}^{\text {in }} \tau_{z}<0$ for $q>1$ whatever $s_{z}^{\text {in }}$.
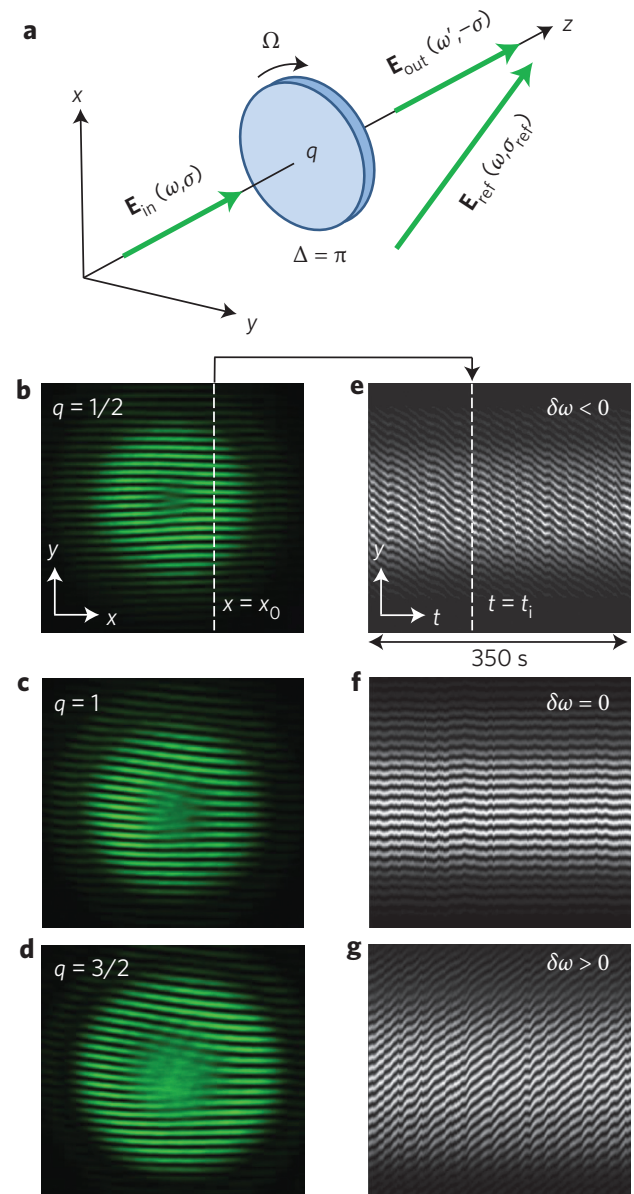

Figure 3 | Demonstration of right-handed, zero and left-handed ORT. a, Schematic of the experiment. A circularly polarized light beam with helicity $\sigma= \pm 1$ and angular frequency $\omega$, labelled $\mathbf{E}_{\mathrm{in}}(\omega, \sigma)$, is normally incident on a sample designed to have a uniform birefringent phase retardation $\Delta=\pi$ at a wavelength of $532 \mathrm{~nm}$. The sample is fixed on a rotating stage whose angular velocity $\Omega$ is externally controlled. The output light field, labelled $\mathbf{E}_{\text {out }}\left(\omega^{\prime}, \sigma\right)$, has frequency $\omega^{\prime}=\omega+\delta \omega$, which may differ from $\omega$, and helicity opposite to that of the input. b-d, Snapshots of the $2 q$-fork intensity patterns $I\left(x, y, t_{i}\right)$ at time $t=t_{i}$ between the output beam and a noncollinear reference beam with helicity $\sigma_{\text {ref }}=\sigma$ and frequency $\omega$ for $q=1 / 2, q=1$ and $q=3 / 2$, respectively. $\mathbf{e}-\mathbf{g}$, Spatiotemporal interferograms taken at $x=x_{0}$, $I\left(x_{0}, y, t\right)$, when $\sigma \Omega>0$ for $q=1 / 2(\mathbf{e}), q=1(\mathbf{f})$ and $q=3 / 2(\mathbf{g})$. The drift direction of the fringing patterns allows the determination of the sign of the rotational Doppler frequency shift, namely $\delta \omega<0, \delta \omega=0$ or $\delta \omega>0$. The wavelength is $532 \mathrm{~nm}$ and $\Omega=0.37$ rad s ${ }^{1}$.

In the context of previous experimental studies on light induced rotation of optically isotropic transparent non axisymmetric micro objects, we note that a Gaussian beam has been shown to lead to either clockwise only or anticlockwise only spinning motion inde pendently of the incident angular momentum ${ }^{26,27}$, and therefore cannot be considered as a left handed optomechanical manifestation. This is also the case for the reversed orbiting motion of a micropar ticle with irregular shape induced by a linearly polarized vortex beam (reported in ref. 28), because the observed sense of rotation is inde pendent of the sign of the incident angular momentum, and also for the polarization dependent rotational motion of an achiral micro particle with well controlled shape induced by a polarized vortex beam (reported in ref. 29), where the observed sense of rotation is given by the sign of the incident angular momentum, hence corre sponding to a right handed ORT. On the other hand, in the case of optically induced torque driven by absorption only, the sign of the 

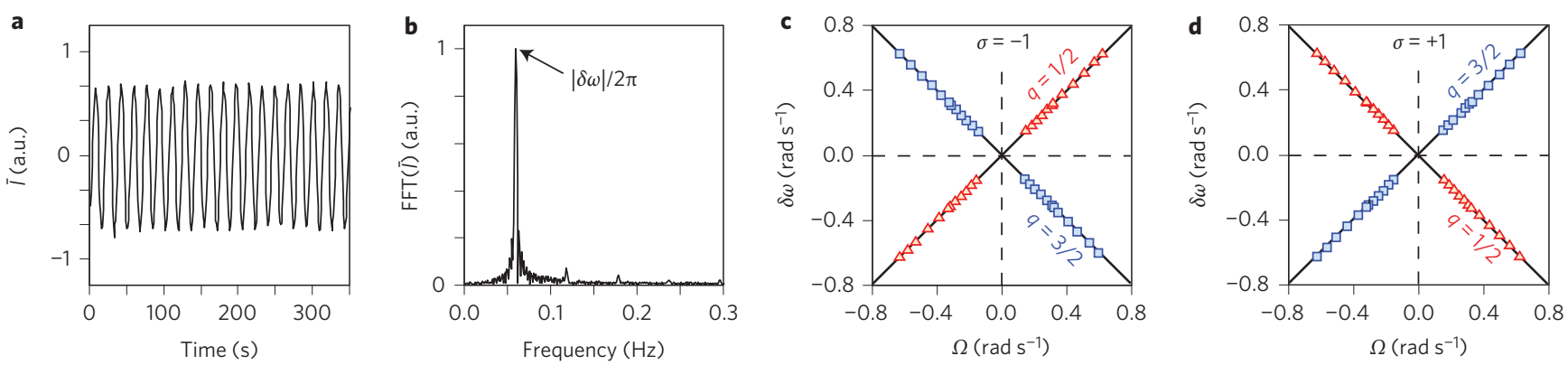

Figure 4 | Quantitative analysis of rotational Doppler experiments. a, Time-oscillating signal $\bar{l}=\int I\left(x_{0}, y, t\right) \mathrm{d} y-\left\langle\int I\left(x_{0}, y, t\right) \mathrm{d} y\right\rangle_{t}$, where $I$ refers to the intensity interferogram pattern shown in Fig. 3 and $\langle\cdot\rangle_{t}$ refers to time averaging. b. Fast Fourier transform (FFT) spectrum of $\bar{l}$, which exhibits a well-defined peak at frequency $|\delta \omega| / 2 \pi$. Here $\Omega=0.37 \mathrm{rad} \mathrm{s}^{1}$ and the wavelength is $532 \mathrm{~nm}$. $\mathbf{c}, \mathbf{d}$, Rotational Doppler angular frequency shift $\delta \omega=\omega^{\prime} \quad \omega$ as a function of the rotating slab angular velocity $\Omega$ for $q=1 / 2$ and $q=3 / 2$ when $\sigma=1$ (c) and $\sigma=+1$ (d). Solid lines show the theoretical results given by equation (2).
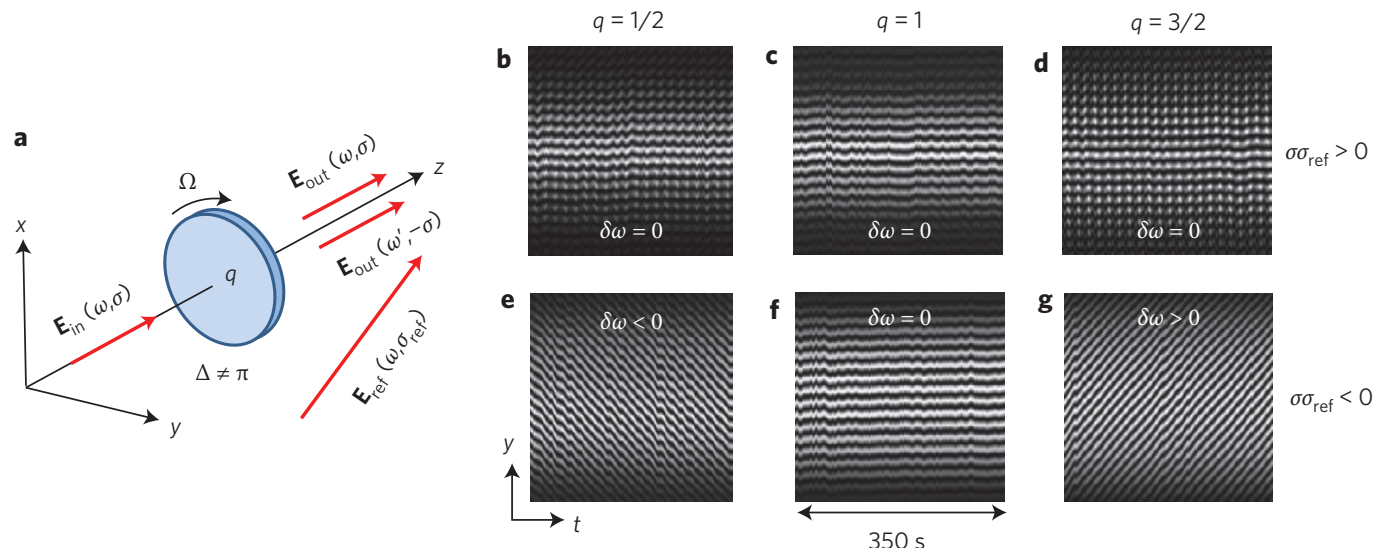

Figure 5 | Generalized ORT rotational Doppler experiments. a, Schematic of the experiment when the uniform birefringent phase delay of the nanostructured glass slab is $\Delta(\omega) \neq \pi$. This leads to an elliptically polarized output light beam that consists of the coherent superposition of two fields with opposite helicities, $\mathbf{E}_{\text {out }}(\omega, \sigma)$ and $\mathbf{E}_{\text {out }}\left(\omega^{\prime}, \sigma\right)$, whose angular frequencies and relative intensity weights may differ. $\mathbf{b}-\mathbf{g}$, The contributions of the output light field components to ORT are retrieved from their respective spatiotemporal interferograms using a non-collinear reference beam with helicity $\sigma_{\text {ref }}= \pm \sigma$ and frequency $\omega$, here shown for $\sigma \Omega>0$ : in the case $\sigma \sigma_{\text {ref }}>0$ for $q=1 / 2(\mathbf{b}), q=1(\mathbf{c})$ and $q=3 / 2(\mathbf{d})$, and in the case $\sigma \sigma_{\text {ref }}<0$ for $q=1 / 2(\mathbf{e}), q=1(\mathbf{f})$ and $q=3 / 2(\mathbf{g})$. Here, the wavelength is $632.8 \mathrm{~nm}$, so $\Delta=0.84 \pi$ and $\Omega=0.37 \mathrm{rad} \mathrm{s}$.

torque is always the same as that of the incident angular momentum, whatever the polarization state and orbital content of the incident light field (shown in ref. 30), which precludes a left handed effect. In the general case where absorption and/or birefringence and/or scattering can be at play, the circumstances under which a left handed rotational effect takes place certainly deserve further study.

By addressing the rotational degree of freedom, the present study strengthens the emergence of a novel field in optical trapping and micromanipulation left handed optomechanics driven by spin orbit scattering of light. As already emphasized for the case of negative optical forces, a left handed optomechanical toolbox may find application in the metrology of fluidic and soft matter systems ${ }^{11,12,14}$. Original optical micro/nanostructuring of matter could also be envisioned for photonics applications. More generally, this study addresses the interaction of light with structured media and is likely to lead to new developments in the science and appli cations of structured light.

\section{Methods}

The three samples with $q=(1 / 2,1,3 / 2)$ were form-birefringent nanostructured glass slabs from Altechna R\&D. The azimuthal distribution of their optical axis is shown to satisfy $\psi=q \phi$, where $\phi$ is the polar angle in the plane of the slab. The glass substrates had a diameter of $1 \mathrm{inch}$, a thickness of $3 \mathrm{~mm}$, and the structured area corresponded to a 4 -mm-diameter region centred on the substrate. The samples were designed to exhibit a uniform birefringent phase retardation over the whole structured area, which equalled $\pi$ at a wavelength of $532 \mathrm{~nm}$. The polariscopic characterization of the samples was carried out using incoherent illumination at
$532 \mathrm{~nm}$ provided by a halogen lamp, in front of which were placed tracing paper and an interference filter (wavelength of $532 \mathrm{~nm}$ ) with $10 \mathrm{~nm}$ spectral width (Thorlabs). The rotational Doppler experiments were performed using either a diode-pumped solid-state laser (Cobolt) operating at $532 \mathrm{~nm}$ or a $\mathrm{He} \mathrm{Ne}$ gas laser (Melles Griot) operating at $632.8 \mathrm{~nm}$. Both laser sources operated on the fundamental TEM $\mathrm{To}_{00}$ Gaussian mode. The incident light beam impinged at normal incidence onto the slab (and was centred on it), and was collimated into a $\sim 2$-mm-diameter beam (at $1 / e^{2}$ of its maximum intensity) using a homemade optical telescope made of a pair of planoconvex lenses whose focal lenses were adapted to the laser source used. The slabs were placed in a rotating holder whose angular velocity was controlled by a voltagecontrolled d.c. motor. The interference intensity patterns were recorded using a CMOS video camera (Thorlabs) and the videos were processed using homemade Matlab code. In all experiments the polarization state of the light was ensured using either linear or circular polarizers (Meadowlark Optics).

\section{References}

1. Grier, D. G. A revolution in optical manipulation. Nature 424, 810816 (2003).

2. Padgett, M. \& Bowman, R. Tweezers with a twist. Nature Photon. 5, 343348 (2011)

3. Lee, S.-H., Roichman, Y. \& Grier, D. G. Optical solenoid beams. Opt. Express 18, 69886993 (2010).

4. Sukhov, S. \& Dogariu, A. On the concept of 'tractor beams'. Opt. Lett. 35, 38473849 (2010).

5. Salandrino A. \& Christodoulides, D. N. Reverse optical forces in negative index dielectric waveguide arrays. Opt. Lett. 36, 31033105 (2011).

6. Chen, J., Ng, J., Lin, Z. \& Chan, C. T. Optical pulling force. Nature Photon. 5, 531534 (2011). 
7. Sukhov, S. \& Dogariu, A. Negative nonconservative forces: optical "tractor beams” for arbitrary objects. Phys. Rev. Lett. 107, 203602 (2011).

8. Novitsky, A., Qiu, C.-W. \& Wang, H. Single gradientless light beam drags particles as tractor beams. Phys. Rev. Lett. 107, 203601 (2011).

9. Nemirovsky, J., Rechtsman, M. C. \& Segev, M. Negative radiation pressure and negative effective refractive index via dielectric birefringence. Opt. Express 20, 89078914 (2012).

10. Novitsky, A., Qiu, C.-W. \& Lavrinenko, A. Material-independent and size-independent tractor beams for dipole objects. Phys. Rev. Lett. 109, 023902 (2012).

11. Dogariu, A., Sukhov, S. \& Saenz, J. Optically induced 'negative forces'. Nature Photon. 7, 2427 (2013).

12. Brzobohatý, O. et al. Experimental demonstration of optical transport, sorting and self-arrangement using a 'tractor beam'. Nature Photon. 7, 123127 (2013).

13. Wang, N., Chen, J., Liu, S. \& Lin, Z. Dynamical and phase-diagram study on stable optical pulling force in Bessel beams. Phys. Rev. A 87, 063812 (2013).

14. Kajorndejnukul, V., Ding, W., Sukhov, S., Qiu, C.-W. \& Dogariu, A. Linear momentum increase and negative optical forces at dielectric interface. Nature Photon. 7, 787790 (2013).

15. Allen, P. J. A radiation torque experiment. Am. J. Phys. 34, 11851192 (1966)

16. Garetz, B. A. Angular Doppler effect. J. Opt. Soc. Am. 71, 609611 (1981).

17. Marston, P. L. Axial radiation force of a Bessel beam on a sphere and direction reversal of the force. J. Acoust. Soc. Am. 120, 3518 (2006).

18. Marston, P. L. Negative axial radiation forces on solid spheres and shells in a Bessel beam. J. Acoust. Soc. Am. 122, 3162 (2007).

19. Mitri, F. G. Negative axial radiation force on a fluid and elastic spheres illuminated by a high-order Bessel beam of progressive waves. J. Phys. A 42, 245202 (2009).

20. Friese, M. E. J., Nieminen, T. A., Heckenberg, N. R. \& Rubinsztein-Dunlop, H Optical torque controlled by elliptical polarization. Opt. Lett. 23, 13 (1998).

21. Beth, R. A. Mechanical detection and measurement of the angular momentum of light. Phys. Rev. 50, 115125 (1936).

22. Friese, M. E. J., Nieminen, T. A., Heckenberg, N. R. \& Rubinsztein-Dunlop, H. Optical alignment and spinning of laser-trapped microscopic particles. Nature 394, 348350 (1998).
23. Marrucci, L., Manzo, C. \& Paparo, D. Optical spin-to-orbital angular momentum conversion in inhomogeneous anisotropic media. Phys. Rev. Lett. 96, 163905 (2006)

24. Shimotsuma, Y., Kazansky, P. G., Qiu, J. \& Hirao, K. Self-organized nanogratings in glass irradiated by ultrashort light pulses. Phys. Rev. Lett. 91, 247405 (2003).

25. Beresna, M., Gecevičius, M., Kazansky, P. G. \& Gertus, T. Radially polarized optical vortex converter created by femtosecond laser nanostructuring of glass. Appl. Phys. Lett. 98, 201101 (2011).

26. Higurashi, E., Ukita, H., Tanaka, H. \& Ohguchi, O. Optically induced rotation of anisotropic micro-objects fabricated by surface micromachining. Appl. Phys. Lett. 64, 22092210 (1994).

27. Galajda, P. \& Ormos, P. Rotation of microscopic propellers in laser tweezers. J. Opt. B 4, S78 S81 (2002).

28. Jesacher, A., Fürhapter, S., Maurer, C., Bernet, S. \& Ritsch-Marte, M. Reverse orbiting of microparticles in optical vortices. Opt. Lett. 31, 28242826 (2006).

29. Asavei, T. et al. Optical angular momentum transfer to microrotors fabricated by two-photon photopolymerization. New J. Phys. 11, 093021 (2009).

30. Friese, M. E. J., Enger, J., Rubinsztein-Dunlop, H. \& Heckenberg, N. R. Optical angular-momentum transfer to trapped absorbing particles. Phys. Rev. A 54, 15931596 (1996)

\section{Acknowledgements}

The authors thank C. Loussert for the polariscopic optical characterization of the samples. This study received financial support from the French State in the frame of the 'Investments for the future' Programme IdEx Bordeaux (reference ANR-10-IDEX-03-02).

\section{Author contributions}

D.H. realized the experimental set-up and conducted the experiments. E.B. conceived the experiment and supervised the project. D.H. and E.B. wrote the manuscript. 